\title{
Visual field effects in the discrimination of sine-wave gratings
}

\author{
FREDERICK L. KITTERLE and LINDA M. SELIG \\ University of Toledo, Toledo, Ohio
}

\begin{abstract}
The time needed to decide whether the second of two successively presented sinusoidal gratings was of a higher or lower spatial frequency than the first was measured for spatial frequencies of $1,2,4,8$, and 12 cycles per degree (cpd) presented in either the left visual field (LVF) or right visual field (RVF). A LVF advantage was found for discriminating within the low-spatialfrequency range (i.e., 1 and 2 cpd), whereas a RVF advantage was found for discriminating within the high-spatial-frequency range (i.e., 4-12 cpd). These findings support the conclusion that hemispheric asymmetries in the processing of gratings arise when comparisons are made between the output of spatial-frequency channels.
\end{abstract}

The perceptual quality of the visual stimulus (i.e., degree of blur, contrast, exposure duration, etc.) is an important factor in determining the magnitude and direction of hemispheric asymmetries (see Sergent, 1983, 1985). The reason perceptual factors play such a role in laterality research is because they influence the way in which stimuli are encoded and represented by the nervous system. It is this representation of visual information that is utilized by higher level cognitive processes. Sergent (1982) has argued that hemispheric asymmetries arise at this higher cognitive level and result from differences between the two cerebral hemispheres in the utilization of spatialfrequency information. The left hemisphere (LH) is hypothesized to be more efficient in the processing of high spatial frequencies, whereas the right hemisphere (RH) is hypothesized to be more efficient in the processing of low spatial frequencies (see Christman, 1989; Kitterle, 1986; Kitterle \& Christman, 1991; Sergent, 1983, 1987; Sergent \& Hellige, 1986, for reviews of this literature). This has come to be called the "spatial-frequency hypothesis." Input factors determine the spatial frequencies available in the stimulus representation, and the nature of the cognitive task determines the spatial frequencies required for effective performance (e.g., Sergent, 1985).

Recent research on the identification of sinusoidal gratings supports this hypothesis. The hemispheric asymmetry is manifested in a double dissociation, that is, in the interaction between spatial frequency and visual field. Reaction time to identify a low-spatial-frequency $(1$ cycle per degree [cpd]) grating was faster for left visual field/right hemisphere (LVF/RH) than for right visual field/left hemisphere (RVF/LH) presentations (Kitterle, Hellige, \& Christman, 1990). On the other hand, time to identify a high-spatial-frequency $(9 \mathrm{cpd})$ grating was faster for

This research was supported by an Academic Challenge Grant from the State of Ohio to enhance research in experimental psychology. Correspondence may be addressed to Frederick L. Kitterle, Department of Psychology, University of Toledo, Toledo, OH 43606.
RVF/LH than for LVF/RH presentations. This finding also holds for the identification of low- and high-spatialfrequency compound gratings (i.e., gratings composed of two or more sine-wave components chosen from the lowor high-spatial-frequency range, respectively) (Christman, Kitterle, \& Hellige, in press). On the other hand, the spatial frequency $\times$ visual field interaction is not found when the task is detection, that is, when subjects are required to depress a key as quickly as possible when a grating is presented irrespective of the spatial frequency of the grating (Kitterle, Christman, \& Hellige, 1990; Kitterle \& Kaye, 1985). In this case, hemispheric symmetry is found. Reaction times to detect a grating are similar for RVF and LVF presentations regardless of spatial frequency.

The finding of a spatial frequency $\times$ visual field interaction for sine-wave grating identification but not for detection may be due to differences in the nature of the decision processes involved. In the case of identification, a comparison between the outputs of spatial-frequency channels is necessary; this is not the case for detection (see Thomas, 1985; Thomas, Gille, \& Barker, 1982). Thus, if a critical component of the computational process necessary for revealing a spatial frequency $\times$ visual field interaction is a comparison of the outputs of spatialfrequency channels, then one might also expect to find this interaction in a grating discrimination task. Based on the spatial-frequency hypothesis, the time to discriminate one low-spatial-frequency grating from another is expected to be faster for LVF presentations, whereas the time to discriminate one high-spatial-frequency grating from another is expected to be quicker for RVF presentations. The present experiment is designed to test this prediction.

\section{METHOD}

\section{Subjects}

Eight right-handed males with normal or corrected-to-normal vision participated in this experiment for course credit. All were naive about the purpose of this study. 


\section{Apparatus and Stimuli}

The stimuli used in these experiments were vertically oriented sinewave gratings that were produced by means of a computer-controlled Picasso CRT Spatio-Temporal Image Synthesizer (Innisfree) and displayed on Tektronix 608 monitors (P-31 phosphor). A large black matte surround subtending $30^{\circ} \times 36^{\circ}$ at a viewing distance of 42 in. was placed directly in front of the monitors. Holes in the surround were used to mask the monitor screens down to two $6.8^{\circ}$ circular displays. A small red fixation point was placed between the two CRTs, and the inner edge of each screen was $3^{\circ}$ from fixation. Signals to the $x$-and $y$-axes of both monitors produced uniformly illuminated screens with a mean luminance of $10.3 \mathrm{~cd} / \mathrm{m}^{2}$.

Signals to the $z$-axis of the monitors produced the various spatial frequencies. The amplitude of these signals controlled the contrast defined as $\left(L_{\max }-L_{\min }\right) /\left(L_{\max }+L_{\min }\right)$. The contrast of the gratings was $20 \%$, and the gratings were exposed for $100 \mathrm{msec}$. Since contrast was modulated about the mean luminance of the screens, there was no change in level of light adaptation during the presentation of the gratings, which were abruptly turned on and off. Both contrast and mean luminances were measured with a Tektronix J16 photometer/radiometer. Care was taken throughout the study to ensure that the monitors remained matched in mean luminance and that the contrast calibrations did not drift.

Five base spatial frequencies $(1,2,4,8$, and 12 cpd) were used. For each base, there was an associated comparison stimulus that differed from the base by \pm .5 octave. The comparison stimulus was presented $100 \mathrm{msec}$ after the offset of the base. To minimize the use of cues other than spatial frequency for discrimination, the contrast of the comparison grating was randomly varied over a $10 \%$ range. To avoid the use of local luminance cues as a basis of discrimination, both the phase of the first and second gratings was randomly varied over trials.

The experiment was carried out in a darkened room. A chin- and headrest was used to provide stable viewing and to aid in maintaining fixation. Viewing was binocular.

\section{Procedure}

Subjects were light adapted to the mean luminance of the monitor screens for $3 \mathrm{~min}$. Following this, they engaged in a series of 24 practice trials. After the practice session, the experiment proper began. On a given trial there was a brief $(200-\mathrm{msec})$ tone, followed at a randomly chosen foreperiod $(500,700$, or $900 \mathrm{msec})$ by a 100 -msec presentation of the base stimulus, a 100 -msec delay, and then a 100-msec presentation of the comparison stimulus. Subjects were instructed to depress one key if the second stimulus was higher in spatial frequency than the first and another key if it was lower in spatial frequency. Half of the subjects responded higher with the left key and lower with the right, and for the other half this was reversed. Subjects were instructed to respond as quickly and accurately as possible. There was a 2 -sec interval between trials. There were 90 replications for each base spatial frequency ( 45 per visual field). The five base spatial frequencies as well as the visual field were randomized across trials within a block.

\section{RESULTS}

The results of this experiment are based on the means of the median reaction time (RT) for each condition for each subject collapsed over hand of response because there was neither a main effect or interaction of hand of response with spatial frequency, visual field, or the interaction of spatial frequency and visual field. Figure 1 RT is plotted as a function of spatial frequency for LVF and RVF presentations. As can be seen in this figure, discriminations are faster for LVF presentations for 1 and 2 cpd gratings.

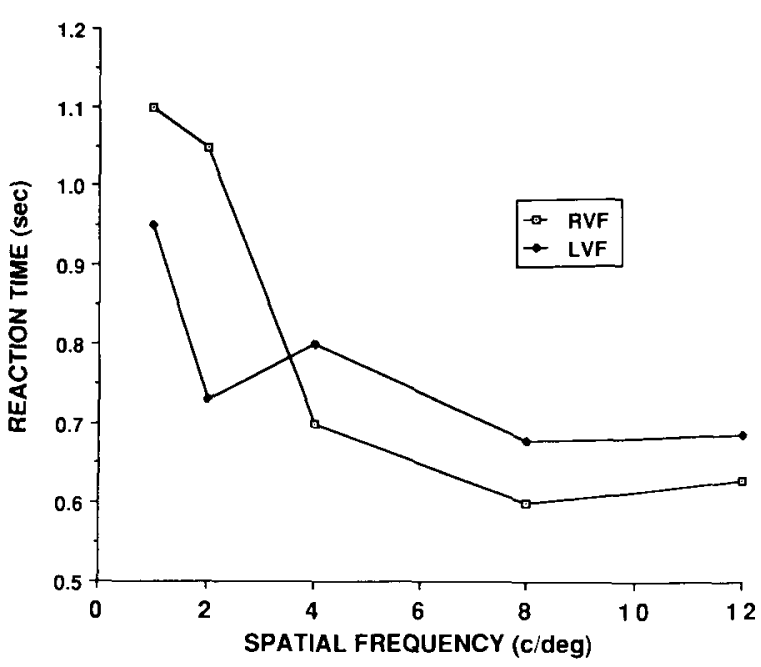

Figure 1. Choice reaction time to discriminate two sinusoidal gratings that differ by .5 octave as a function of spatial frequency for LVF/RH (open symbols) and RVF/LH (filled symbols).

However, with increasing spatial frequency there is a shift. Here, RVF presentations yield faster reaction times. A 5 (spatial frequency) $\times 2$ (visual field) repeated measures analysis of variance verifies the existence of a significant spatial frequency $\times$ visual field interaction $[F(4,28)=$ $4.27, p<.01]$. The effects of spatial frequency are also significant $[F(4,28)=13.79, p<.001]$. The time needed to discriminate two gratings decreased with increases in base spatial frequency. However, the effect of visual field was not significant $[F(1,7)<1]$.

\section{DISCUSSION}

The results of the present experiment support the spatialfrequency hypothesis and earlier conclusions that hemispheric asymmetries with gratings may arise in tasks that involve comparisons among the outputs of spatial-frequency channels, that is, discrimination and identification of spatial frequency.

These results, however, contrast with other gratingdiscrimination studies that have failed to find a hemisphere $X$ spatial frequency interaction with normal (Fiorentini \& Berardi, 1984; Szelag, Budohoska, \& Koltuska, 1987) and clinical (Grabowska, Semenza, Denes, \& Testa, 1989) populations. Kitterle, Hellige, and Christman (1990) pointed out that this could have arisen as a result of failures to control for changes in mean luminance that accompanied grating presentations (i.e., Grabowska et al., 1989; Szelag et al., 1987). Changes in mean luminance can have complex effects on the processing of spatial frequencies (Badcock \& Sevdalis, 1987; Green, 1981; Kitterle, Corwin, \& Berta, 1979).

Fiorentini and Berardi (1984) controlled for changes in mean luminance and measured the minimum spatialfrequency difference that could be detected from a base 
stimulus of $1 \mathrm{cpd}$ over a range of contrasts. They did not find any consistent evidence for differences between the hemispheres in spatial-frequency thresholds, although one subject showed a small LVF advantage. Since Fiorentini and Berardi (1984) used only one spatial frequency, it is not possible to test for a spatial frequency $\times$ visual field interaction. However, based on the results of the present study, it is possible that a more sensitive measure of hemispheric-processing efficiency is the time to process spatial-frequency information. In this case, an indirect indicator of processing time could be revealed by RT measures, as the results of Figure 1 suggest.

There are other, more subtle, effects that may have mitigated against the finding of hemispheric asymmetries in the processing of spatial frequency. In our experiment, we randomly varied the phase of both the base and comparison gratings over trials to prevent subjects from using local luminance cues as a basis for discrimination. This was not the case for Grabowska et al. (1989) or Szelag et al. (1987). It is possible that subjects could have based their discrimination on whether the local luminance maxima or minima in the two flashes coincided rather than on the spatial frequency per se. Alternatively, they could have based the discrimination on a comparison of the distance of a local luminance maxima (minima) from the edge of the display for each of the two successive flashes.

In the case of studies using square-wave gratings, discrimination could have been based not only on the fundamental frequency but also alternately on the higher harmonics. For example, the width of a bar of one grating might be compared with the width of another, or the comparison might be made between the distance of one sharp edge from another. In the former case, low-spatialfrequency information is used, whereas in the latter, highspatial-frequency information would form the basis of the discrimination. If these putative strategies shifted over trials, hemispheric effects might have cancelled out. The idea that task demands can direct attention to either lowor high-spatial-frequency information in a square-wave grating that leads to shifts in hemispheric dominance has been demonstrated by Kitterle, Hellige, and Christman (1990). They have shown that discriminations based on the fundamental frequency of a square-wave grating yields a LVF advantage, whereas attention to the edges yields a RVF advantage.

In most of the discrimination studies, the time elapsed between the presentation of the first stimulus and the offset of the second has been long enough for eye movements and/or shifts of attention to occur. Since eye movements were not monitored, it is quite possible that they may have accounted for the failure to find any evidence for a spatial frequency $\times$ visual field interaction even though a more sensitive measure, RT, was used. For example, in Szelag et al. (1987), the total time was greater than $1 \mathrm{sec}$ (i.e., 40-msec exposure of each grating pattern and a 1.5 -sec interstimulus interval). Although eye movements were not monitored in the present experiment, we attempted to minimize their effect by keeping the total presentation time of the stimulus display relatively short and strongly emphasized to subjects that to perform the discrimination task accurately, it was necessary to maintain fixation.

Finally, we note that unlike simple reaction time experiments where RT increases with spatial frequency, our results show that for discrimination, RT decreased with increases in spatial frequency for both visual fields. Greenlee and Breitmeyer (1989) showed that with foveal presentations, different functions describe changes in simple RT and discrimination RT with spatial frequency. The former monotonically increased with spatial frequency, while the latter was an inverted $U$-shaped function of spatial frequency. Slower RTs were found for spatial-frequency discriminations around $2-3 \mathrm{cpd}$, that is, in the spatialfrequency region where sensitivity is the highest. Greenlee and Breitmeyer (1989) suggested that spatial-frequency channels may be more dense in the mid-spatial-frequency range than at higher or lower frequencies, which would account for greater sensitivity. However, as a result of the greater density and overlap of channels in this region, more pairwise comparisons would be required for discrimination. Thus, the longer reaction times at intermediate spatial frequencies are the result of a relatively longer serial comparison process than at higher or lower spatial frequencies. Our results suggest that at greater eccentricities, there is a shift in the density of channels toward the lower spatial frequencies. Moreover, the fact that the slopes for the RVF and LVF differ may imply hemispheric differences in the nature of the comparison or differences between the two hemispheres in the speed of serial comparisons among spatial-frequency channels.

\section{REFERENCES}

Badcock, D. R., \& SEvdalis, E. (1987). Masking by uniform field flicker: Some practical considerations. Perception, 15, 641-647.

Christman, S. (1989). Perceptual characteristics in visual laterality research. Brain \& Cognition, 11, 238-257.

Christman, S., Krtterle, F. L., \& Heluge, J. (in press). Hemispheric asymmetry in the processing of absolute versus relative spatial frequency. Brain \& Cognition.

FIORENTINI, A., \& BERARDI, N. (1984). Right-hemisphere superiority in the discrimination of spatial phase. Perception, 13, 695-708.

Grabowska, A., Semenza, C., Denes, G., a Testa, S. (1989). Impaired grating discrimination following right hemisphere damage. Neuropsychologia, 27, 259-263.

GreEN, M. A. (1981). Spatial frequency effects in masking by light. Vision Research, 21, 861-866.

Greenlee, M. W., \& Breitmeyer, B. (1989). A choice reaction time analysis of spatial frequency discrimination. Vision Research, 11, 1575-1586.

KitTerle, F. L. (1986). Psychophysics of lateral tachistoscopic presentation. Brain \& Cognition, 5, 131-162.

Kitterle, F. L., Christman, S. (1991). Hemispheric symmetries and asymmetries in processing spatial frequency. In F. L. Kitterle (Ed.), Cerebral laterality: Theory and research: The Toledo symposium (pp. 201-224). Hillsdale, NJ: Erlbaum.

Kitterle, F. L., Christman, S., Hellige, J. (1990). Hemispheric differences are found in the identification, but not the detection, of low versus high spatial frequencies. Perception \& Psychophysics, 48, 297-306.

Kitterle, F. L., Corwin, T. R., \& Berta, J. (1979). Masking of 
sinusoidal targets by uniform fields of unequal duration. Journal of the Optical Society of America, 69, 1445.

Kitterle, F. L., Hellige, J., \& Christman, S. (1990). Hemispheric differences in the processing of sine- and square-wave gratings. Manuscript in preparation.

KITterLe, F., \& KAYE, R. S. (1985). Hemispheric symmetry in contrast and orientation sensitivity. Perception \& Psychophysics, 37, 391-396.

SeRgent, J. (1982). The cerebral balance of power: Confrontation or cooperation? Journal of Experimental Psychology: Human Perception \& Performance, $8,253-272$.

SERGENT, J. (1983). Role of input in visual hemispheric asymmetries. Psychological Bulletin, 93, 481-512.

SERGENT, J. (1985). Influence of task and input factors on hemispheric involvement in face processing. Journal of Experimental Psychology: Human Perception \& Performance, 11, 846-861.
SERGENT, J. (1987). Failures to confirm the spatial-frequency hypothesis: Fatal blow or healthy complication? Canadian Journal of Psychology, 41, 412-428.

Sergent, J., \& Hellige, J. (1986). Role of input factors in visual-field asymmetries. Brain \& Cognition, 5, 174-199.

SzelaG, E., Budohoska, W., \& Koltuska, B. (1987). Hemispheric differences in the perception of gratings. Bulletin of the Psychonomic Society, 25, 95-98.

Thomas, J. (1985). Detection and identification: How are they related? Journal of the Optical Society of America A, 2, 1457-1467.

Thomas, J., Gille, J., \& Barker, R. (1982). Simultaneous visual detection and identification: Theory and data. Joumal of the Optical Society of America, 72, 1642-1651.

(Manuscript received April 4, 1990;

revision accepted for publication February 20, 1991.) 